\title{
A Comparative Study on the Stability of Laplace-Adomian Algorithm and Numerical Methods in Generalized Pantograph Equations
}

\author{
Sabir Widatalla ${ }^{1,2}$ \\ ${ }^{1}$ Department of Mathematics, Harbin Institute of Technology, Harbin 150001, China \\ ${ }^{2}$ Department of Mathematics, Sinnar University, Singa 107, Sudan
}

Correspondence should be addressed to Sabir Widatalla, sabirtag@yahoo.com

Received 31 May 2012; Accepted 29 July 2012

Academic Editors: T. Allahviranloo and K. T. Miura

Copyright () 2012 Sabir Widatalla. This is an open access article distributed under the Creative Commons Attribution License, which permits unrestricted use, distribution, and reproduction in any medium, provided the original work is properly cited.

The main objective of this paper is to examine the stability and convergence of the Laplace-Adomian algorithm to approximate solutions of the pantograph-type differential equations with multiple delays. This is done by comparatively investigating it with other methods.

\section{Introduction}

Delay differential equations (DDEs) are a large and important class of differential equations in which the derivative of the unknown function at a certain time is given in terms of the values of the function at previous times. They often arise in wide and diverse range of applications in population studies [1,2], economics [3], medical biology $[4,5]$, controls of mechanical systems $[6]$, and so forth. The pantograph equation is one of the most important kinds of DDEs. The name pantograph was used by Iserles and Liu [7] to study how the electric current is collected by the pantograph of an electric locomotive, from where it gets its name.

In recent years, some promising approximate analytical solutions have been proposed, such as the Taylor collocation method [8], Bernstein polynomials [9], spline method [10, $11]$, and other methods are reviewed in $[2,12,13]$.

In this work we consider the following problems.

\section{Problem 1.}

$$
\begin{gathered}
u^{\prime}(t)=\beta u(t)+f\left(t, u(t), u\left(q_{1} t\right), u\left(q_{2} t\right), \ldots, u\left(q_{\ell} t\right)\right), \\
u(0)=u_{0} .
\end{gathered}
$$

Problem 2.

$$
\begin{gathered}
u^{(m)}(t)=f\left(t, u(t), u\left(q_{1} t\right), u\left(q_{2} t\right), \ldots, u\left(q_{\ell} t\right)\right), \\
\sum_{k=0}^{m-1} C_{i k} u^{(k)}(0)=\lambda_{i}, \quad i=0,1, \ldots, m-1,
\end{gathered}
$$

where $f$ is an analytical function, $C_{i k}, \lambda_{i}$, and $\beta \in \ldots C ; 0<$ $q_{i}<1, i=1,2, \ldots, \ell$.

The aim of this paper is to employ and examine the stability of the Laplace-Adomian algorithm (LAA) for solving Problems 1 and 2. This method was first proposed by Khuri [14], who applied the scheme to a class of nonlinear differential equations. In this method the solution is given as an infinite series usually converging very rapidly to the exact solution of the problem.

A major advantage of this method is that it is free from round-off errors and without any discretization or restrictive assumptions. Therefore, results obtained by LAA are more accurate and efficient. LAA has been shown to accurately and easily approximate solutions of large class of linear and nonlinear ODEs and PDEs [14-16]; for example, Ongun [17] employed LAA to give an approximate solution of 
nonlinear ordinary differential equation systems, such as a model for HIV infection of $\mathrm{CD}^{+} \mathrm{T}$ cells, Wazwaz [18] also used this method for handling the nonlinear Volterra integro-differential equations, Khan and Faraz [19] modified LAA to obtain series solutions of the boundary layer equation, and Yusufoglu [20] adapted LAA to solve the Duffing equation.

The numerical technique of LAA basically illustrates how the Laplace transforms are used to approximate the solution of the nonlinear differential equations by manipulating the decomposition method that was first introduced by Adomian $[21,22]$.

\section{Laplace-Adomian Algorithm}

To illustrate the basic idea of the Laplace-Adomian algorithm, we consider the following nonlinear operator:

$$
u^{\prime}(t)=R u+N u+f(t)
$$

with the initial condition

$$
u(0)=u_{0},
$$

where $R$ is a linear operator, $N$ is a nonlinear operator, and $f(t)$ is a given analytical function.

The technique consists first of applying the Laplace transform (denoted throughout this paper by $\mathcal{L}$ ) to both sides of (3), to get

$$
\mathcal{L}\left[u^{\prime}(t)\right]=\mathcal{L}[R u]+\mathcal{L}[N u]+\mathcal{L}[f(t)]
$$

Applying the formulas of the Laplace transform, we obtain

$$
\mathcal{L}[u(t)]=\mathscr{H}(s)+\frac{1}{s} \mathcal{L}[R u]+\frac{1}{s} \mathcal{L}[N u]
$$

where

$$
\mathscr{H}(s)=\frac{1}{s}(u(0)+\mathcal{L}[f(t)]) .
$$

Suppose the answer to (3) is as follows:

$$
u(t)=\sum_{n=0}^{\infty} u_{n}(t)
$$

where the terms $u_{n}(t)$ are to be recursively computed, the nonlinear operator $\mathrm{Nu}$ is decomposed as follows:

$$
N u(t)=\sum_{n=0}^{\infty} A_{n}
$$

where $A_{n}$ is an infinite series of the Adomian polynomials $u_{0}, u_{1}, \ldots, u_{n}$, calculated by the formula [21]

$$
A_{n}=\frac{1}{n !} \frac{d^{n}}{d \lambda^{n}}\left[N\left(\sum_{i=0}^{n} \lambda^{i} u_{i}\right)\right]_{\lambda=0}, \quad n=0,1,2, \ldots
$$

Substituting (8) and (9) into (6) leads to

$$
\mathcal{L}\left[\sum_{n=0}^{\infty} u_{n}(t)\right]=\mathcal{H}(s)+\frac{1}{s} \mathcal{L}\left[R \sum_{n=0}^{\infty} u_{n}\right]+\frac{1}{s} \mathcal{L}\left[\sum_{n=0}^{\infty} A_{n}\right] .
$$
as

Using the linearity of the Laplace transform gives (11)

$$
\sum_{n=0}^{\infty} \mathcal{L}\left[u_{n}(t)\right]=\mathscr{H}(s)+\frac{1}{s} \sum_{n=0}^{\infty} \mathcal{L}\left[R u_{n}\right]+\frac{1}{s} \sum_{n=0}^{\infty} \mathcal{L}\left[A_{n}\right]
$$

Matching both sides of (11) yields

$$
\begin{gathered}
\mathcal{L}\left[u_{0}(t)\right]=\frac{1}{s}(u(0)+\mathcal{L}[f(t)])=\mathcal{H}(s), \\
\mathcal{L}\left[u_{1}(t)\right]=\frac{1}{s} \mathcal{L}\left[R u_{0}\right]+\frac{1}{s} \mathcal{L}\left[A_{0}\right], \\
\mathcal{L}\left[u_{2}(t)\right]=\frac{1}{s} \mathcal{L}\left[R u_{1}\right]+\frac{1}{s} \mathcal{L}\left[A_{1}\right] .
\end{gathered}
$$

Generally

$$
\mathcal{L}\left[u_{n+1}(t)\right]=\frac{1}{s} \mathcal{L}\left[R u_{n}\right]+\frac{1}{s} \mathcal{L}\left[A_{n}\right], \quad n \geq 0 .
$$

Applying the inverse Laplace transform to (13) gives the initial approximation

$$
u_{0}(t)=\mathcal{L}^{-1}\left[\frac{1}{s}(u(0)+\mathcal{L}[f(t)])\right]=H(t)
$$

Substituting this value of $u_{0}$ into the inverse Laplace transform of (14) gives $u_{1}$. The other terms $u_{2}, u_{3}, \ldots$ can be obtained recursively in similar fashion from

$$
u_{n+1}(t)=\mathcal{L}^{-1}\left[\frac{1}{s} \mathcal{L}\left[R u_{n}\right]+\frac{1}{s} \mathcal{L}\left[A_{n}\right]\right], \quad n \geq 0 .
$$


Using (18), we can rewrite them as follows to obtain $u$ :

$$
\begin{aligned}
& u_{0}=\mathcal{L}^{-1}\left[\frac{1}{s}(u(0)+\mathcal{L}[f(t)])\right]=H(t), \\
& u_{1}=\mathcal{L}^{-1}\left[\frac{1}{s} \mathcal{L}\left[R u_{0}\right]+\frac{1}{s} \mathcal{L}\left[A_{0}\right]\right] \\
& =\left(\mathcal{L}^{-1} \mathcal{s}^{-1} \mathcal{L} R\right) u_{0}+\left(\mathcal{L}^{-1} s^{-1} \mathcal{L}\right) A_{0} \\
& u_{2}=\mathcal{L}^{-1}\left[\frac{1}{s} \mathcal{L}\left[R u_{1}\right]+\frac{1}{s} \mathcal{L}\left[A_{1}\right]\right] \\
& =\mathcal{L}^{-1}\left[s^{-1} \mathcal{L}\left[R\left(\left(\mathcal{L}^{-1} s^{-1} \mathcal{L} R\right) u_{0}+\left(\mathcal{L}^{-1} s^{-1} \mathcal{L}\right) A_{0}\right)\right]\right. \\
& \left.+s^{-1} \mathcal{L}\left[A_{1}\right]\right] \\
& =\left(\mathcal{L}^{-1} s^{-1} \mathcal{L} R\right)^{2} u_{0}+\left(\mathcal{L}^{-1} s^{-1} \mathcal{L} R\right)\left(\mathcal{L}^{-1} s^{-1} \mathcal{L}\right) A_{0} \\
& +\left(\mathcal{L}^{-1} s^{-1} \mathcal{L}\right) A_{1} \\
& u_{3}=\mathcal{L}^{-1}\left[\frac{1}{s} \mathcal{L}\left[R u_{2}\right]+\frac{1}{s} \mathcal{L}\left[A_{2}\right]\right] \\
& =\left(\mathcal{L}^{-1} s^{-1} \mathcal{L} R\right)^{3} u_{0}+\left(\mathcal{L}^{-1} s^{-1} \mathcal{L} R\right)^{2}\left(\mathcal{L}^{-1} s^{-1} \mathcal{L}\right) A_{0} \\
& +\left(\mathcal{L}^{-1} \mathcal{s}^{-1} \mathcal{L} R\right)\left(\mathcal{L}^{-1} \mathcal{s}^{-1} \mathcal{L}\right) A_{1}+\left(\mathcal{L}^{-1} \mathcal{s}^{-1} \mathcal{L}\right) A_{2}, \\
& u_{n+1}=\mathcal{L}^{-1}\left[\frac{1}{s} \mathcal{L}\left[R u_{n}\right]+\frac{1}{s} \mathcal{L}\left[A_{n}\right]\right] \\
& =\left(\mathcal{L}^{-1} s^{-1} \mathcal{L} R\right)^{n+1} u_{0}+\left(\mathcal{L}^{-1} s^{-1} \mathcal{L} R\right)^{n}\left(\mathcal{L}^{-1} \boldsymbol{s}^{-1} \mathcal{L}\right) A_{0} \\
& +\left(\mathcal{L}^{-1} \mathcal{s}^{-1} \mathcal{L} R\right)^{n-1}\left(\mathcal{L}^{-1} \mathcal{s}^{-1} \mathcal{L}\right) A_{1} \\
& +\cdots+\left(\mathcal{L}^{-1} s^{-1} \mathcal{L}\right) A_{n},
\end{aligned}
$$

Subsiutiting the values of $u_{0}, u_{1}, u_{2}, \ldots$ into (8) gives

$$
\begin{aligned}
u(t)= & H(t)+\left(\mathcal{L}^{-1} s^{-1} \mathcal{L} R\right) u_{0}+\left(\mathcal{L}^{-1} s^{-1} \mathcal{L}\right) A_{0} \\
& +\left(\mathcal{L}^{-1} s^{-1} \mathcal{L} R\right)^{2} u_{0} \\
& +\left(\mathcal{L}^{-1} s^{-1} \mathcal{L} R\right)\left(\mathcal{L}^{-1} s^{-1} \mathcal{L}\right) A_{0} \\
& +\cdots+\left(\mathcal{L}^{-1} s^{-1} \mathcal{L} R\right)^{n} u_{0}+\left(\mathcal{L}^{-1} s^{-1} \mathcal{L} R\right)^{n-1} \\
& \times\left(\mathcal{L}^{-1} s^{-1} \mathcal{L}\right) A_{0}+\left(\mathcal{L}^{-1} s^{-1} \mathcal{L} R\right)^{n-2}\left(\mathcal{L}^{-1} s^{-1} \mathcal{L}\right) A_{1} \\
& +\cdots+\left(\mathcal{L}^{-1} s^{-1} \mathcal{L}\right) A_{n}+\cdots \\
= & \sum_{m=0}^{\infty}\left(\mathcal{L}^{-1} s^{-1} \mathcal{L} R\right)^{m} H(t) \\
& +\sum_{m=1}^{\infty} \sum_{k=0}^{m-1}\left(\mathcal{L}^{-1} s^{-1} \mathcal{L} R\right)^{m-k-1}\left(\mathcal{L}^{-1} s^{-1} \mathcal{L}\right) A_{k} .
\end{aligned}
$$

The sum of $m$ terms $u_{0}, u_{1}, u_{2}, \ldots, u_{m-1}$ in (8), that is, $Q_{m}=\sum_{n=0}^{m-1} u_{n}$, where $m \rightarrow \infty, Q_{m}$ tends to $u$. This means that $Q_{m}$ is an appropriate approximation of $u$. The terms in the above series soon tend to zero where $1 /(m n)$ ! has been the coefficients of calculations derived from the operation $\left(\mathcal{L}^{-1} \mathcal{s}^{-1} \mathcal{L}\right), m$ is the number of terms, and $n$ is the order of the operation derivation. Therefore, it has a rapid convergence.

\section{Test Problems}

In this section, we will apply Laplace-Adomian algorithm to solve the pantograph delay equation. The absolute errors in Tables $1-4$ are the values of $\left|u(t)-\sum_{i=0}^{n} u_{i}(t)\right|$ at selected points. All iterates are calculated by using Matlab 7.

3.1. Stability of the Laplace-Adomian Algorithm. A method is said to be stable when the obtained solution undergoes small variations as there are slight variations in inputs and parameters and when probable perturbations in parameters that are effective in equations and conditions prevailing them do not introduce, in comparison to the physical reality of the problem, any perturbations in what is returned. We propose here to compare the Laplace-Adomian algorithm with other numerical methods by offering examples and examining the stability of the Laplace-Adomian algorithm.

Example 1 (Example (Evans and Raslan [13])). Consider the following pantograph equation:

$$
u^{\prime}(t)=\frac{1}{2} u(t)+\frac{1}{2} e^{(1 / 2) t} u\left(\frac{t}{2}\right), \quad 0 \leq t \leq 1, u(0)=1
$$

The exact solution is $u(t)=e^{t}$. Applying the result of (17) gives us

$$
u_{0}(t)=\mathscr{L}^{-1}\left[\frac{1}{s}\right]=1
$$

The iteration formula (18) for this example is

$$
u_{n+1}(t)=\mathcal{L}^{-1}\left[\frac{1}{2 s} \mathcal{L}\left[u_{n}+e^{(t / 2) t} u_{n}\left(\frac{t}{2}\right)\right]\right], \quad n \geq 0
$$

In Table 1 we make a comparison between the absolute errors obtained by the Bernstien series [9] in column 2, together with the spline method $[10,11]$ in columns 3 and 4 , and finally, the Laplace-Adomian algorithm with $n=$ 5,6 , and 7 in the last three columns. Given the following absolute errors that were obtained from these methods, we can conclude that the rate of convergence of LAA is higher than the other methods. However, taking a closer look at the errors of LAA shows that the error increases significantly more than the other methods in the bottom of the columns. This means that the stability of LAA is decreasing more than the other methods. 
TABle 1: Comparison of the absolute errors for Example 1.

\begin{tabular}{|c|c|c|c|c|c|c|}
\hline \multirow{2}{*}{$t$} & \multirow{2}{*}{$\begin{array}{l}\text { Bernstein series [9] } \\
\qquad N=7\end{array}$} & \multicolumn{2}{|c|}{ Spline method, $h=0.001$} & \multicolumn{3}{|c|}{ Present method } \\
\hline & & $m=2[10]$ & $m=3[11]$ & $n=5$ & $n=6$ & $n=7$ \\
\hline 0.2 & $0.3004 E-8$ & $0.198 E-7$ & $1.37 E-11$ & $6.737 E-9$ & $9.726 E-11$ & $1.220 E-12$ \\
\hline 0.4 & $0.3610 E-8$ & $0.473 E-7$ & $3.27 E-11$ & $4.530 E-7$ & $1.301 E-8$ & $3.250 E-10$ \\
\hline 0.6 & $0.4024 E-8$ & $0.847 E-7$ & $5.86 E-11$ & $5.428 E-6$ & $2.326 E-7$ & $8.675 E-9$ \\
\hline 0.8 & $0.4161 E-8$ & $0.135 E-6$ & $9.54 E-11$ & $3.212 E-5$ & $1.825 E-6$ & $9.033 E-8$ \\
\hline 1.0 & $0.8934 E-7$ & $0.201 E-6$ & $1.43 E-10$ & $1.292 E-4$ & $9.125 E-6$ & $5.617 E-7$ \\
\hline
\end{tabular}

TABLE 2: Comparison of the absolute errors for $q=0.2$ for Example 2 .

\begin{tabular}{|c|c|c|c|c|c|}
\hline \multirow{2}{*}{$t$} & \multicolumn{2}{|c|}{ Taylor collocation method [8] } & \multicolumn{3}{|c|}{ Present method } \\
\hline & $N=10$ & $N=12$ & $n=2$ & $n=3$ & $n=4$ \\
\hline $2^{-1}$ & $0.200 E-9$ & $0.124 E-9$ & $1.600 E-2$ & $2.052 E-3$ & $2.088 E-4$ \\
\hline $2^{-2}$ & $0.100 E-9$ & $0.974 E-10$ & $2.137 E-3$ & $1.353 E-4$ & $6.823 E-6$ \\
\hline $2^{-3}$ & $0.100 E-9$ & $0.700 E-10$ & $2.763 E-4$ & $8.686 E-6$ & $2.181 E-7$ \\
\hline $2^{-4}$ & $0.100 E-9$ & $0.914 E-10$ & $3.513 E-5$ & $5.504 E-7$ & $6.915 E-9$ \\
\hline $2^{-5}$ & $0.100 E-9$ & $0.528 E-10$ & $4.430 E-6$ & $3.464 E-8$ & $2.684 E-10$ \\
\hline $2^{-6}$ & 0.000 & $0.195 E-10$ & $5.561 E-7$ & $2.172 E-9$ & $7.417 E-11$ \\
\hline
\end{tabular}

Example 2 (Example (Muroya et al. [23])). Consider the following pantograph equation:

$$
u^{\prime}(t)=-u(t)+\frac{q}{2} u(q t)-\frac{q}{2} e^{-q t}, \quad 0 \leq t \leq 1, u(0)=1 .
$$

The exact solution is $u(t)=e^{-t}$. Applying the result of (17), gives us

$$
u_{0}(t)=\mathcal{L}^{-1}\left[\frac{1}{s}\left(1-\mathcal{L}\left[\frac{q}{2} e^{-q t}\right]\right)\right]=\frac{1}{2}+\frac{1}{2} e^{-q t} .
$$

The other terms of the sequences $u_{n}$ can be obtained directly from (18) as in general

$$
u_{n+1}(t)=\mathcal{L}^{-1}\left[\frac{1}{s} \mathcal{L}\left[-u_{n}+\frac{q}{2} u_{n}(q t)\right]\right], \quad n \geq 0
$$

Table 2 compares the results of the Laplace-Adomian algorithm and the Taylor collocation method [8]. It can be seen that the Laplace-Adomian algorithm is weaker in the stability than Taylor collocation method and yet is stronger in the convergence.

Example 3 (Example (Liu and Li [12])). Consider the multipantograph delay equation with variable coefficients

$$
\begin{array}{r}
u^{\prime}(t)=-u(t)+\mu_{1}(t) u(0.5 t)+\mu_{2}(t) u(0.25 t), \\
0 \leq t \leq 1, u(0)=1 .
\end{array}
$$

Here $\mu_{1}(t)=-e^{-0.5 t} \sin (0.5 t)$, and $\mu_{2}(t)=-2 e^{-0.75 t} \cos$ $(0.5 t) \sin (0.25 t)$. The initial approximation for this example is

$$
u_{0}(t)=\mathcal{L}^{-1}\left[\frac{1}{s}\right]=1
$$

And from (18), we obtain

$$
\begin{aligned}
u_{n+1}(t) & \\
=\mathcal{L}^{-1}\left(\frac{1}{s} \mathcal{L}[\right. & -u_{n}-e^{-(1 / 2) t} \sin \left(\frac{t}{2}\right) u_{n}\left(\frac{t}{2}\right) \\
& \left.\left.-2 e^{-(3 / 4) t} \cos \left(\frac{t}{2}\right) \sin \left(\frac{t}{4}\right) u_{n}\left(\frac{t}{4}\right)\right]\right), \quad n \geq 0 .
\end{aligned}
$$

Comparisons of approximate solutions for few terms with exact solution $u(t)=e^{-t} \cos (t)$ are illustrated in Table 3 .

Example 4. Consider the pantograph equation of third order

$$
\begin{array}{r}
u^{\prime}(t)=1-\sin (t)-2 u^{2}\left(\frac{t}{2}\right)+2 \cos \left(\frac{t}{2}\right) u\left(\frac{t}{2}\right) \\
0 \leq t \leq 1, u(0)=0
\end{array}
$$

Let us start with an initial approximation

$$
u_{0}(t)=\mathcal{L}^{-1}\left[\frac{1}{s} \mathcal{L}[1-\sin (t)]\right]=t-1+\cos (t)
$$

The other terms of the sequences $u_{n}$ can be obtained directly from (18)

$$
u_{n+1}(t)=\mathcal{L}^{-1}\left[\frac{2}{s} \mathcal{L}\left[-A_{n}\left(\frac{t}{2}\right)+\cos \left(\frac{t}{2}\right) u_{n}\left(\frac{t}{2}\right)\right]\right], \quad n \geq 0,
$$


TABle 3: Absolute errors for Example 3.

\begin{tabular}{lcccc}
\hline$t$ & Exact value & \multicolumn{3}{c}{ The present method } \\
$n=2$ & $1.404 E-3$ & $n=3$ \\
\hline 0.2 & $8.0241 E-1$ & $2.080 E-2$ & $1.164 E-2$ & $7.026 E-5$ \\
0.4 & $6.1740 E-1$ & $8.483 E-2$ & $4.018 E-2$ & $6.169 E-3$ \\
0.6 & $4.5295 E-1$ & $1.918 E-1$ & $9.639 E-2$ & $1.964 E-2$ \\
0.8 & $3.1305 E-1$ & $3.387 E-1$ & $1.889 E-1$ & $4.851 E-2$ \\
1.0 & $1.9876 E-1$ & $5.206 E-1$ & & \\
\hline
\end{tabular}

TABle 4: Absolute errors for Example 4.

\begin{tabular}{lcccc}
\hline$t$ & Exact value & \multicolumn{3}{c}{ The present method } \\
$n=1$ & $4.655 E-6$ & $2.134 E-8$ \\
\hline 0.2 & $1.9866 E-1$ & $5.524 E-4$ & $2.313 E-5$ & $2.240 E-6$ \\
0.4 & $3.8941 E-1$ & $3.548 E-3$ & $1.063 E-4$ & $2.572 E-5$ \\
0.6 & $5.6464 E-1$ & $9.196 E-3$ & $9.314 E-4$ & $1.334 E-4$ \\
0.8 & $7.1735 E-1$ & $1.562 E-2$ & $3.470 E-3$ & $4.523 E-4$ \\
1.0 & $8.4147 E-1$ & $1.931 E-2$ & & \\
\hline
\end{tabular}

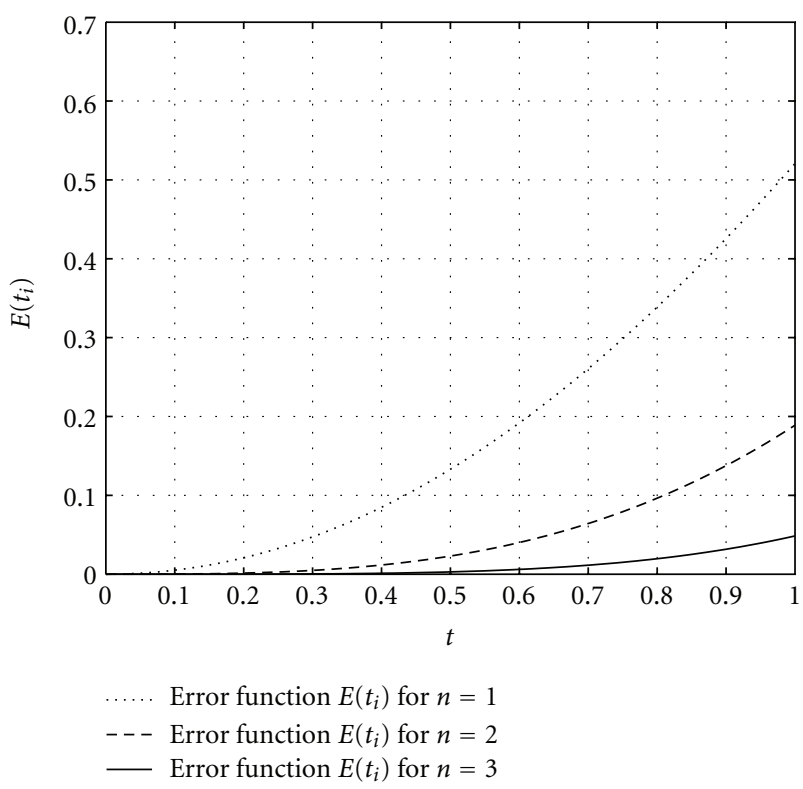

FIGURE 1: Error $E\left(t_{i}\right)$ for $n=1,2$ and 3 for Example 3.

where $A_{n}$ is the Adomian polynomials that represent the nonlinear term $u^{2}(x / 2)$. The Adomian polynomials for $A(u(t))=u^{2}(t / 2)$ are given by

$$
\begin{aligned}
A_{0} & =u_{0}^{2}\left(\frac{t}{2}\right) \\
A_{1} & =2 u_{0}\left(\frac{t}{2}\right) u_{1}\left(\frac{t}{2}\right), \\
A_{2} & =u_{1}^{2}\left(\frac{t}{2}\right)+2 u_{0}\left(\frac{t}{2}\right) u_{2}\left(\frac{t}{2}\right), \\
& \vdots
\end{aligned}
$$

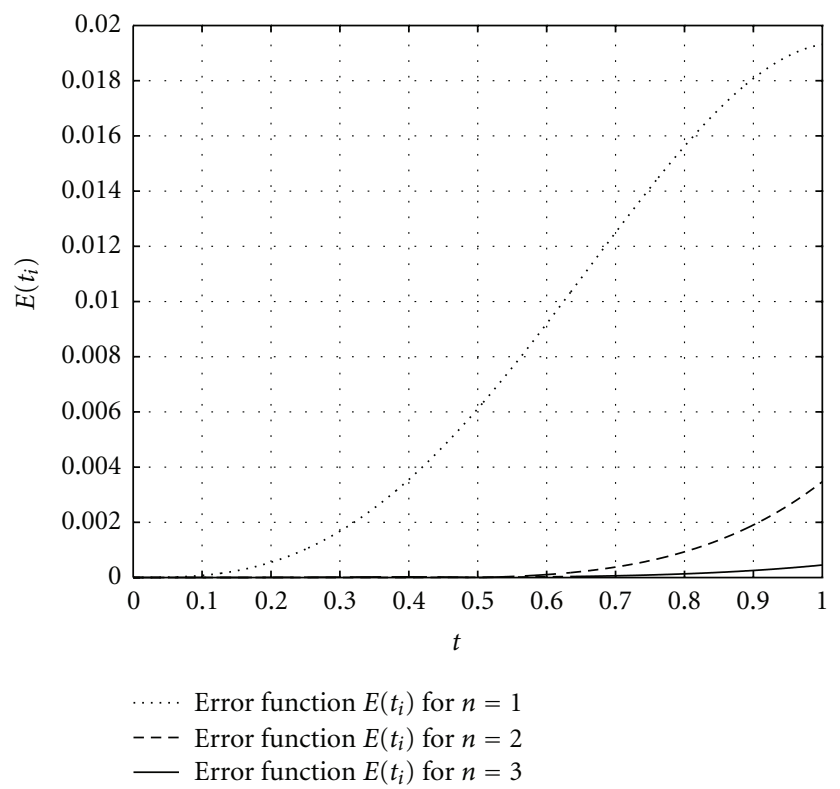

Figure 2: Error $E\left(t_{i}\right)$ for $n=1,2$ and 3 for Example 4.

Table 4 shows the absolute error of the numerical approximation by using a few iterations.

The results for Examples 3-4 in Figures 1 and 2, respectively, show that the approximations converge rapidly and only a few iterations are sufficient to obtain accurate solutions.

Example 5. Consider the multipantograph equation

$$
\begin{array}{r}
u^{\prime}(t)=-\frac{5}{6} u(t)+4 u\left(\frac{t}{2}\right)+9 u\left(\frac{t}{3}\right)+t^{2}-1 \\
0 \leq t \leq 1, u(0)=1
\end{array}
$$


Following the procedures in the previous examples gives us

$$
u_{0}(t)=\mathcal{L}^{-1}\left[\frac{1}{s}+\frac{2}{s^{4}}-\frac{1}{s^{2}}\right]=1-t+\frac{t^{3}}{3} .
$$

According to (18), we obtain

$$
\begin{gathered}
u_{1}(t)=\frac{73}{6} t-\frac{25}{12} t^{2}, \\
u_{2}(t)=\frac{1825}{72} t^{2}-\frac{175}{216} t^{3}, \\
u_{3}(t)=\frac{12775}{1296} t^{3}, \\
u_{n}(t)=0, \quad \forall n \geq 4 .
\end{gathered}
$$

Thus

$$
u(t)=\sum_{n=0}^{\infty} u_{n}(t)=1+\frac{67}{6} t+\frac{1675}{72} t^{2}+\frac{12157}{1296} t^{3}
$$

which is the exact solution.

\section{Conclusion}

In this paper, the Laplace-Adomian algorithm has been successfully applied to the generalized pantograph equations with multiple delays to obtain high approximate solutions or exact solutions with little iterations used. Moreover, the Laplace-Adomian algorithm is simple and easy to use and stronger in convergence compared with other methods. Despite these advantages, it is seen that its stability is lower than other numerical methods.

\section{References}

[1] W. G. Aiello, H. I. Freedman, and J. Wu, "Analysis of a model representing stage-structured population growth with statedependent time delay," SIAM Journal on Applied Mathematics, vol. 52, no. 3, pp. 855-869, 1992.

[2] Y. Kuang, Delay Differential Equations with Applications in Population Dynamics, Academic, New York, NY, USA, 1993.

[3] P. Brunovský, A. Erdélyi, and H.-O. Walther, "On a model of a currency exchange rate-local stability and periodic solutions," Journal of Dynamics and Differential Equations, vol. 16, no. 2, pp. 393-432, 2004.

[4] R. V. Culshaw and S. Ruan, "A delay-differential equation model of HIV infection of $\mathrm{CD}^{+}$T-cells," Mathematical Biosciences, vol. 165, no. 1, pp. 27-39, 2000.

[5] A. De Gaetano and O. Arino, "Mathematical modelling of the intravenous glucose tolerance test," Journal of Mathematical Biology, vol. 40, no. 2, pp. 136-168, 2000.

[6] H. O. Walther, "On a model for soft landing with statedependent delay," Journal of Dynamics and Differential Equations, vol. 19, no. 3, pp. 593-622, 2007.

[7] A. Iserles and Y. K. Liu, "On pantograph integro-differential equations," Journal of Integral Equations and Applications, vol. 6, no. 2, pp. 213-237, 1994.

[8] M. Gülsu and M. Sezer, "A taylor collocation method for solving high-order linear pantograph equations with linear functional argument," Numerical Methods for Partial Differential Equations, vol. 27, no. 6, pp. 1628-1638, 2011.
[9] O. R. Işik, Z. Güney, and M. Sezer, "Bernstein series solutions of pantograph equations using polynomial interpolation," Journal of Difference Equations and Applications, vol. 18, no. 3, pp. 357-374, 2012.

[10] A. El-Safty and S. M. Abo-Hasha, "On the application of spline functions to initial value problems with retarded argument," International Journal of Computer Mathematics, vol. 32, pp. 173-179, 1990.

[11] M. Shadia, Numerical solution of delay differential and neutral differential equations using spline methods [Ph.D. thesis], Assuit University, Assuit, Egypt, 1992.

[12] M. Z. Liu and D. Li, "Properties of analytic solution and numerical solution of multi-pantograph equation," Applied Mathematics and Computation, vol. 155, no. 3, pp. 853-871, 2004.

[13] D. J. Evans and K. R. Raslan, "The Adomian decomposition method for solving delay differential equation," International Journal of Computer Mathematics, vol. 82, no. 1, pp. 49-54, 2005.

[14] S. A. Khuri, "A Laplace decomposition algorithm applied to a class of nonlinear differential equations," Journal of Applied Mathematics, vol. 1, no. 4, pp. 141-155, 2001.

[15] M. Khan, M. Hussain, H. Jafari, and Y. Khan, "Application of Laplace decomposition method to solve nonlinear coupled partial differential equations," World Applied Sciences Journal, vol. 9, Special Issue of Applied Math, pp. 13-19, 2010.

[16] S. A. Khuri, "A new approach to Bratu's problem," Applied Mathematics and Computation, vol. 147, no. 1, pp. 131-136, 2004.

[17] M. Y. Ongun, "The Laplace Adomian Decomposition Method for solving a model for HIV infection of $\mathrm{CD}^{+} \mathrm{T}$ cells," Mathematical and Computer Modelling, vol. 53, no. 5-6, pp. 597-603, 2011.

[18] A. M. Wazwaz, "The combined Laplace transform-Adomian decomposition method for handling nonlinear Volterra integro-differential equations," Applied Mathematics and Computation, vol. 216, no. 4, pp. 1304-1309, 2010.

[19] Y. Khan and N. Faraz, "Application of modified Laplace decomposition method for solving boundary layer equation," Journal of King Saud University, vol. 23, no. 1, pp. 115-119, 2011.

[20] E. Yusufoglu, "Numerical solution of Duffing equation by the Laplace decomposition algorithm," Applied Mathematics and Computation, vol. 177, no. 2, pp. 572-580, 2006.

[21] G. Adomian, Solving Frontier Problems of Physics: The Decomposition Method, Kluwer Academic Publishers, Boston, Mass, USA, 1994.

[22] G. Adomian, "A review of the decomposition method in applied mathematics," Journal of Mathematical Analysis and Applications, vol. 135, no. 2, pp. 501-544, 1988.

[23] Y. Muroya, E. Ishiwata, and H. Brunner, "On the attainable order of collocation methods for pantograph integrodifferential equations," Journal of Computational and Applied Mathematics, vol. 152, no. 1-2, pp. 347-366, 2003. 


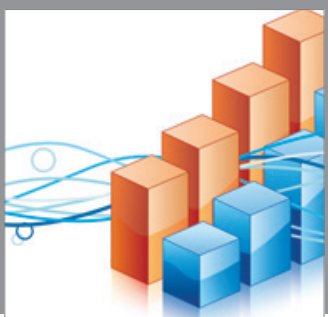

Advances in

Operations Research

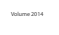

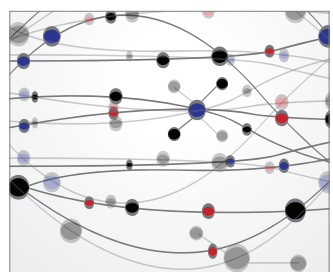

\section{The Scientific} World Journal
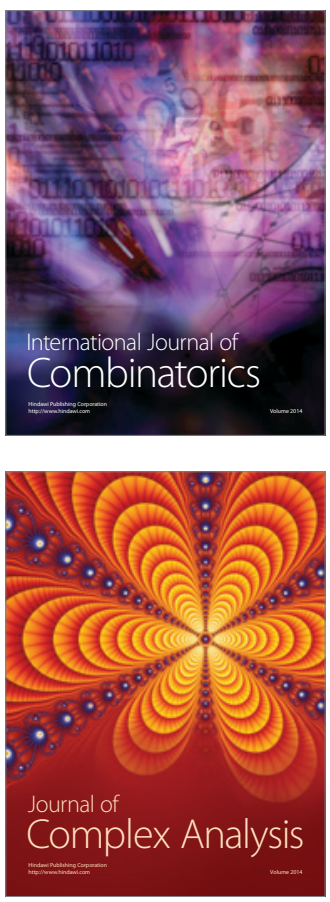

International Journal of

Mathematics and

Mathematical

Sciences
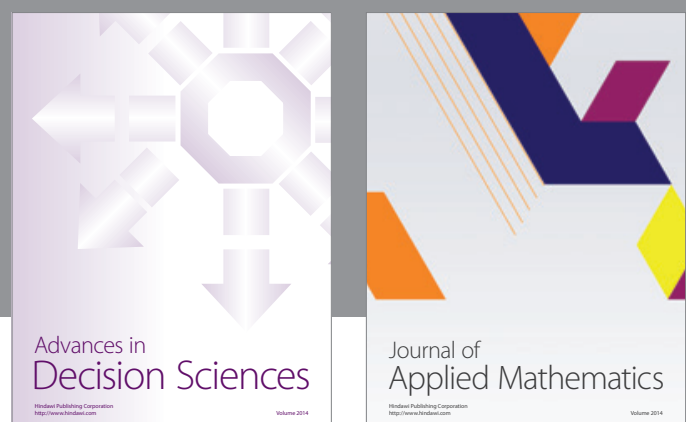

Journal of

Applied Mathematics
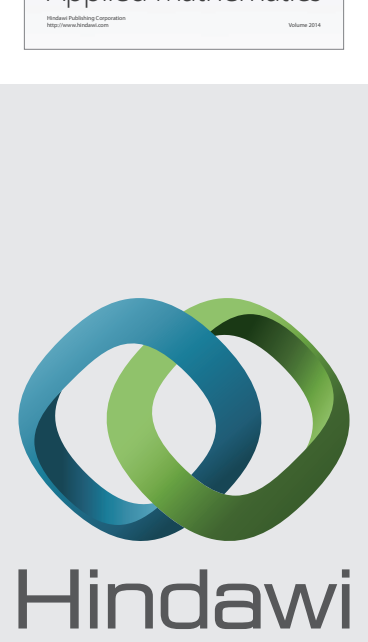

Submit your manuscripts at http://www.hindawi.com
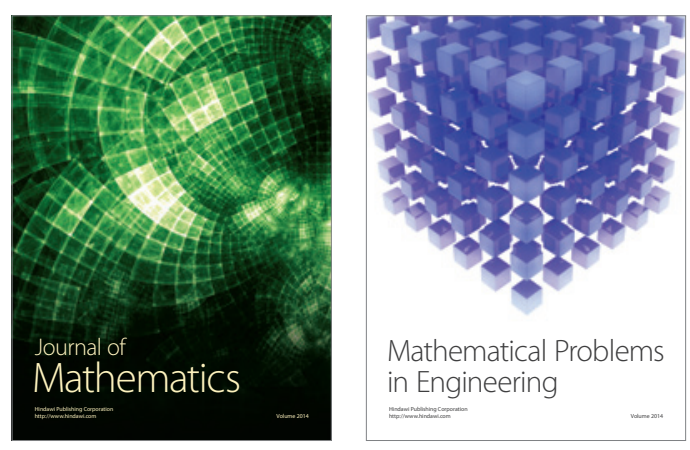

Mathematical Problems in Engineering
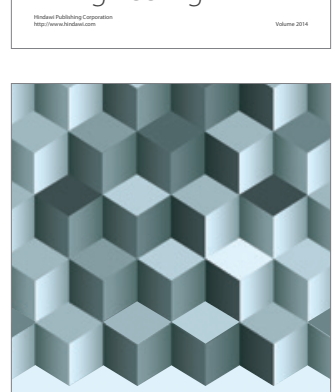

Journal of

Function Spaces
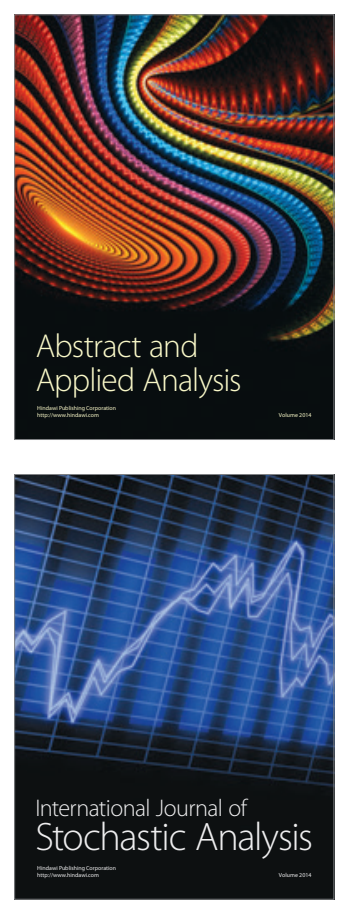

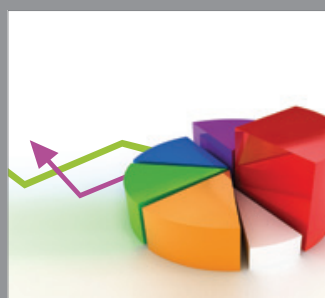

ournal of

Probability and Statistics

Promensencen
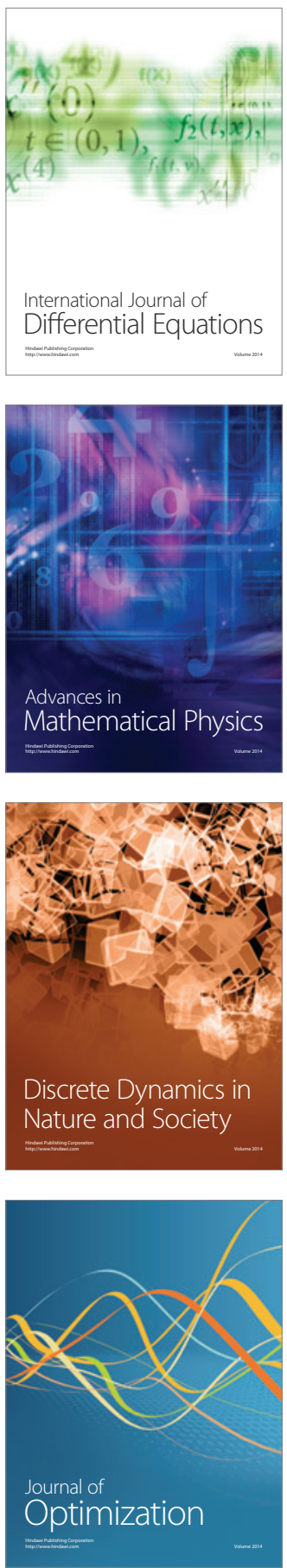